\title{
A novel framework of routing policy for energy-efficient wireless sensor networks: Progress-based nearest forwarding
}

\author{
Sho Sasaki ${ }^{a)}$, Yuichi Miyaji, and Hideyuki Uehara \\ Graduate School of Engineering, Toyohashi University of Technology, \\ 1-1 Hibarigaoka, Tempaku-cho, Toyohashi-shi, Aichi 441-8580, Japan \\ a)sasakis@comm.ee.tut.ac.jp
}

\begin{abstract}
A number of battery-driven sensor nodes are deployed to operate a wireless sensor network, and many routing protocols have been proposed to reduce energy consumption for data communications. This letter proposes a novel routing framework for multihop networks, which is based on transmission power control, nearest forwarding and packet progress toward a sink, in consideration of reducing the extra number of hops in the networks. The energy consumption for the topology employing the proposed forwarding is evaluated by simulation, and it is shown that the proposed scheme is more energy-efficient than the shortest-path tree constructed by Dijkstra's algorithm.
\end{abstract}

Keywords: wireless sensor network, multihop communication, routing, energy efficiency

Classification: Network

\section{References}

[1] B. Karp and H. T. Kung, "GPSR: Greedy perimeter stateless routing for wireless sensor networks," Proc. 6th Annu. Int. Conf. Mobile Comput. and Netw. (MobiCom'00), Boston, MA, pp. 243-254, Aug. 2000. DOI:10.1145/ 345910.345953

[2] S. De, A. Caruso, T. Chaira, and S. Chessa, "Bounds on hop distance in greedy routing approach in wireless ad hoc networks," Int. J. Wireless Mobile Comput., vol. 1, no. 2, pp. 131-140, Feb. 2006. DOI:10.1504/IJWMC.2006. 012472

[3] M. N. Jambli, H. Lenando, K. Zen, S. M. Suhaili, and A. Tully, "Transmission power control in mobile wireless sensor networks: Simulation-based approach," Proc. IET Int. Conf. Wireless Commun. and Applicat. (ICWCA 2012), Kuala Lumpur, Malaysia, pp. 1-6, Oct. 2012. DOI:10.1049/cp.2012. 2109

[4] J. Gomez and A. T. Champbell, "Variable-range transmission power control in wireless ad hoc networks," IEEE Trans. Mobile Comput., vol. 6, no. 1, pp. 87-99, Jan. 2007. DOI:10.1109/TMC.2007.250673

[5] G. Rahmatollahi and G. Abreu, "Closed-form hop-count distributions in 
random networks with arbitrary routing," IEEE Trans. Commun., vol. 60, no. 2, pp. 429-444, Feb. 2012. DOI:10.1109/TCOMM.2012.010512.110125

[6] M. Haenggi, "On routing in random Rayleigh fading networks," IEEE Trans. Wireless Commun., vol. 4, no. 4, pp. 1553-1562, July 2005. DOI:10.1109/ TWC.2005.850376

[7] Q. H. Li, P. Fan, and D. O. Wu, "Energy efficient routing in ad hoc networks with Nakagami-m fading channels," Proc. 2009 Int. Conf. Commun. (ICC 2009), Dresden, Germany, pp. 1-6, June 2009. DOI:10.1109/ICC.2009. 5198992

[8] G. Jacinto, N. Antunes, and A. Pacheco, "Nearest neighbor connectivity in two-dimensional multihop MANETs," in New Advances in Statistical Modeling and Applicat., ed. A. Pacheco, R. Santos, M. do Rosário Oliveira, and C. D. Paulino, pp. 85-94, Springer, Cham, 2014.

[9] E. W. Dijkstra, "A note on two problems in connexion with graphs," Numer. Math., vol. 1, no. 1, pp. 269-271, Dec. 1959. DOI:10.1007/BF01386390

[10] I. Stojmenovic and X. Lin, "Power-aware localized routing in wireless networks," IEEE Trans. Parallel Distrib. Syst., vol. 12, no. 11, pp. 1122-1133, Nov. 2001. DOI:10.1109/71.969123

[11] M. Haenggi, "On distances in uniformly random networks," IEEE Trans. Inf. Theory, vol. 51, no. 10, pp. 3584-3586, Oct. 2005. DOI:10.1109/TIT.2005. 855610

[12] E. Kuiper and S. Nadjm-Tehrani, "Geographical routing with location service in intermittently connected MANETs," IEEE Trans. Veh. Technol., vol. 60, no. 2, pp. 592-604, Feb. 2011. DOI:10.1109/TVT.2010.2091658

[13] W. B. Heinzelman, A. P. Chandrakasan, and H. Balakrishnan, "An applicationspecific protocol architecture for wireless microsensor networks," IEEE Trans. Wireless Commun., vol. 1, no. 4, pp. 660-670, Oct. 2002. DOI:10.1109/TWC. 2002.804190

\section{Introduction}

There have been many routing protocols for wireless ad-hoc/sensor networks in literature. One of the most empirical packet forwarding methods in location-aware protocols is the greedy forwarding, in which a forwarding node can make a locally optimal choice in choosing a next-hop node. The locally optimal choice is the neighbor geographically closest to the destination/sink [1], and thus it is based on packet hop progress toward the destination. This locally optimal choice means that the greedy forwarding is based on the disk model (or protocol model), i.e., each node has the same transmission power and limited common communication range. Although this forwarding policy can minimize the remaining distance to the destination in each hop [2], there occurs the dead-end problem, where a node cannot deliver data to the sink despite the existence of another route which can reach the sink. Moreover, the connectivity between two arbitrary nodes cannot be guaranteed when the network is quite sparse, resulting in the emergence of isolated nodes.

Power saving of sensor nodes is a problem of vital significance for providing beneficial services in wireless sensor networks (WSNs). Routing protocols with (variable-range) transmission power control (TPC) are an alternative choice which aims at guaranteeing both energy efficiency and network connectivity. TPC sets the 
radio transmission power of each node to the right level according to the communication distance, which enables preventing the networks from the dead-end problem. In [3], TPC mechanism is implemented in AODV routing protocol for mobile WSNs, and it is shown that the implementation of TPC has some impact on the networks in respect to energy consumption. In [4], the minimum spanning tree rooted at one source node is constructed on the basis of TPC, and it is clarified that compared to the disk model, the variable-range strategy can save transmission power, as well as improving the traffic capacity.

We can divide routing policies into three types: random-, furthest- and nearestneighbor forwarding. Random-neighbor forwarding is a concept often used to model network processes like distributed localization and flooding, while furthestand nearest-neighbor routing are often design choices for packet hopping/routing [5]. Here, furthest-neighbor forwarding is the same framework as the greedy method referred to above. Nearest-neighbor forwarding is applied to the literature $[6,7,8]$, and it is said to achieve a good performance under energy consideration for most scenarios $[6,7]$.

In this letter, we propose a novel framework of simple packet forwarding strategy. The proposed routing scheme is cherry-picking: the features applied are progress-based routing, variable-range TPC, and nearest-neighbor forwarding. It defines the forwarding angle to search for next-hop relay nodes. Simulation results compare the proposed routing strategy with a shortest-path tree (SPT). The SPT scheme simulated in this letter also performs TPC, but does not follow either nearest-neighbor forwarding or hop progress. Instead, it constructs a tree structure rooted at the sink such that the sum of costs is minimized (to be described later).

\section{Proposed routing policy}

For efficient routing, progress should be made at each hop, i.e., the next-hop neighbor should be closer to the sink [11]. When constructing multihop routes, however, some routing protocols possibly have the hops in which the next-hop node is farther from the sink than the previous node. For instance, SPT-based topologies [10] generally optimize distance-based costs for constructing multihop paths, which may lead to the emergence of regress hops, some of which are to be found in Fig. 1(a). This SPT minimizes the summed cost $\sum_{i=1}^{N_{H}} r_{i}^{\alpha}$ by Dijkstra's algorithm [9], where $r_{i}$ is the $i$-th hop Euclidean distance, $N_{H}$ is the hop-count and $\alpha$ is the path-loss exponent, to connect all nodes in a network to the sink. Consequently, although the energy required for data transmission can be optimized at each path, the number of hops increases, resulting in an inefficient detour routing in terms of energy consumption. Therefore, we propose the progress-based nearest forwarding (PNF) routing as a routing framework for energy-efficient WSNs.

Fig. 1(b) illustrates PNF routing policy. PNF routing considers a neighborhood area toward the sink for each node to search for the next-hop node. The neighborhood area has an angle defined by $\phi$, where $0 \leq \phi<\pi / 2$ to guarantee hop progress. Note that the opening angle of every neighboring sector is $2 \phi$, i.e., the neighboring sector becomes a semi-circle if $\phi=\pi / 2$. Thus, any node located outside the neighborhood area is passed over for the next-hop candidate even if it is the 


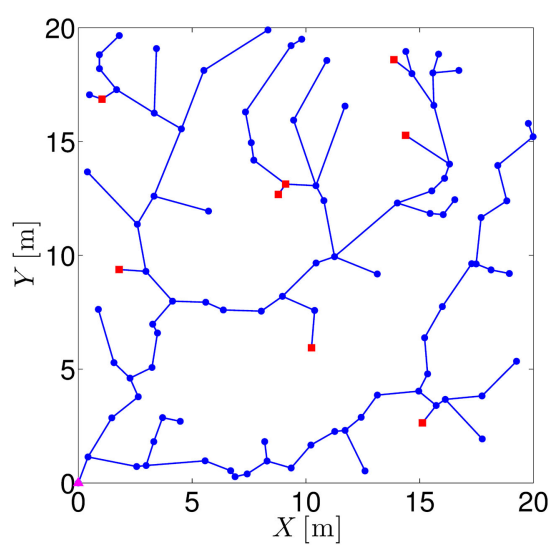

(a) SPT by Dijkstra's algorithm [10]

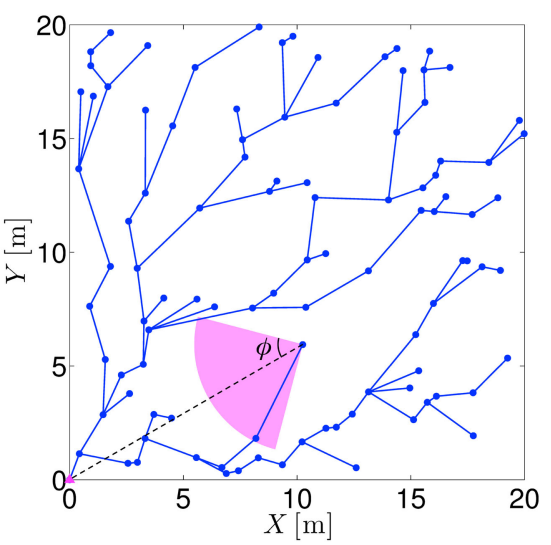

(b) PNF with $\phi=\pi / 4$

Fig. 1. Illustration of routing policies with 100 nodes in a $20 \mathrm{~m} \times 20 \mathrm{~m}$ field. Square markers indicate the nodes whose next-hops regress away from the sink.

nearest from the previous node. Fig. 1(b) shows PNF routing for $\phi=\pi / 4$, which has a fan-shaped sector with an opening angle of $\pi / 2$ toward the sink for each hop. We choose the angle as $\phi=\pi / 4$ for the practical range of neighborhood used in this letter. Assuming that nodes in a network are distributed according to the two-dimensional Poisson point process with intensity $\rho$ [11], the probability density function of hop distance and the expected hop distance in PNF routing are given by

$$
\begin{gathered}
f(r)=2 \rho \phi r e^{-\rho \phi r^{2}}, \\
E[R]=\frac{1}{2} \sqrt{\frac{\pi}{\rho \phi}},
\end{gathered}
$$

respectively. Especially for $\phi=\pi / 4$, the hop distance follows the Rayleigh distribution with $E[R]=1 / \sqrt{\rho}$.

Although following nearest-neighbor forwarding, PNF routing does not immoderately make the number of hops increase. This is because it is based on hop progress and the forwarding angle $\phi$. The smaller number of hops means the longer progress per hop and the smaller latency. (The evaluations on them are not included in this letter.) On the other hand, WSNs with the larger number of hops are likely to have smaller energy consumption per hop due to short hops. We show the energy efficiency for the whole network in simulation results to be described, in terms of whether multihop routing topologies should have the smaller (PNF) or larger (SPT) number of hops.

When it comes to construct location-based packet forwarding topologies such as SPT and PNF, location information on all nodes and the sink is required. It is achieved by measuring the received signal strength from other nodes and the sink, and/or using geographical location service [12]. In addition, considering a locationbased TPC mechanism for routing, overhead energy for channel state estimation has an effect on the total energy consumption. Giving the same number of nodes and the same node arrangement, however, both PNF and SPT routings have the same number of edges (links) in the network, leading to the same additional energy budget for location information retrieving and channel state estimation. Therefore we can have a consequence that the fair premise in both routing policies results in 
an impartial energy addition, and we omit the consideration of those kinds of extra energy budgets from this letter.

\section{Performance evaluations}

We compare the energy consumption of all the nodes in the network for SPT and PNF routing. One sink and $N$ nodes are deployed in a square network area, with the sink at the origin $(0,0)$ and the nodes randomly distributed (see Fig. 1). Each node aggregates data from its children and its own sensed data into one packet, and transmits it to its parent. We set the payload length and the header length as $S=500$ bytes and $H=25$ bytes, respectively. We also set the energy for data communication per bit and data aggregation per bit as $E_{\text {elec }}=50 \mathrm{~nJ} / \mathrm{bit}$ and $E_{\text {agg }}=5 \mathrm{~nJ} / \mathrm{bit}$, respectively. Note that the two-ray ground reflection model is used for radio propagation; therefore, the energy required for data reception $E_{R}$, data sensing/aggregation $E_{A}$ and data transmission $E_{T}$ at each node are calculated as

$$
\begin{aligned}
& E_{R}=\left(N_{d} S+H\right) E_{\text {elec }} \cdot \mathbf{1}\left\{N_{d}>0\right\}, \\
& E_{A}=\left(N_{d}+1\right) S E_{\text {agg }}, \\
& E_{T}= \begin{cases}\left(\left(N_{d}+1\right) S+H\right)\left(E_{\text {elec }}+\varepsilon_{f s} r^{2}\right), & \text { for } r<r_{0}, \\
\left(\left(N_{d}+1\right) S+H\right)\left(E_{\text {elec }}+\varepsilon_{m p} r^{4}\right), & \text { for } r \geq r_{0},\end{cases}
\end{aligned}
$$

where $N_{d}$ is the number of descendent nodes, $\varepsilon_{f s}=10 \mathrm{pJ} / \mathrm{bit} / \mathrm{m}^{2}$ is the free-space propagation loss coefficient, $\varepsilon_{m p}=0.0013 \mathrm{pJ} / \mathrm{bit} / \mathrm{m}^{4}$ is the multipath propagation loss coefficient, $r$ is the transmission distance, and $r_{0}=\sqrt{\varepsilon_{f s} / \varepsilon_{m p}}$ is the break point at critical distance. Also, $\mathbf{1}\{\cdot\}$ denotes the set indicator function. As for energy parameters, we use the energy model in [13] which has been widely adopted to studies in WSNs to determine each type of energy mentioned above. We do not count the extra energy consumption due to overhearing nodes or packet retransmissions, as well as the energy for node localization and channel state estimation, in order to focus on and investigate the energy consumed in the phase of data forwarding. Energy consumption is evaluated over 10,000 independent random realizations.

Fig. 2 illustrates the energy reduction rate $E R R$ [\%],

$$
E R R=\left(1-\frac{E_{\text {total }}^{\mathrm{PNF}}}{E_{\text {total }}^{\mathrm{SPT}}}\right) \times 100,
$$

where $E_{\text {total }}^{\mathrm{SPT}}$ is the energy required for SPT routing, and $E_{\text {total }}^{\mathrm{PNF}}$ is for PNF routing. $E R R$ describes how much energy consumption is reduced in PNF routing compared to SPT. It can be seen from Fig. 2 that PNF routing is more energy-efficient than SPT in all cases of interest. PNF routing reduces energy consumption by at least $8.64 \%$ of SPT, and at most $30.2 \%$. In addition to that, it is noteworthy that the reduction rate is dependent on the node density of the network. Generally, the energy reduction effect is larger as the number of nodes increases. This is due to the larger number of hops in larger-density networks. The energy reduction is attributed mainly to the reduction of the number of hops. Fig. 3(a) and 3(b) show the maximum number of hops in the network and the total energy consumption for SPT and PNF routing, respectively. It can be seen that although the number of hops 


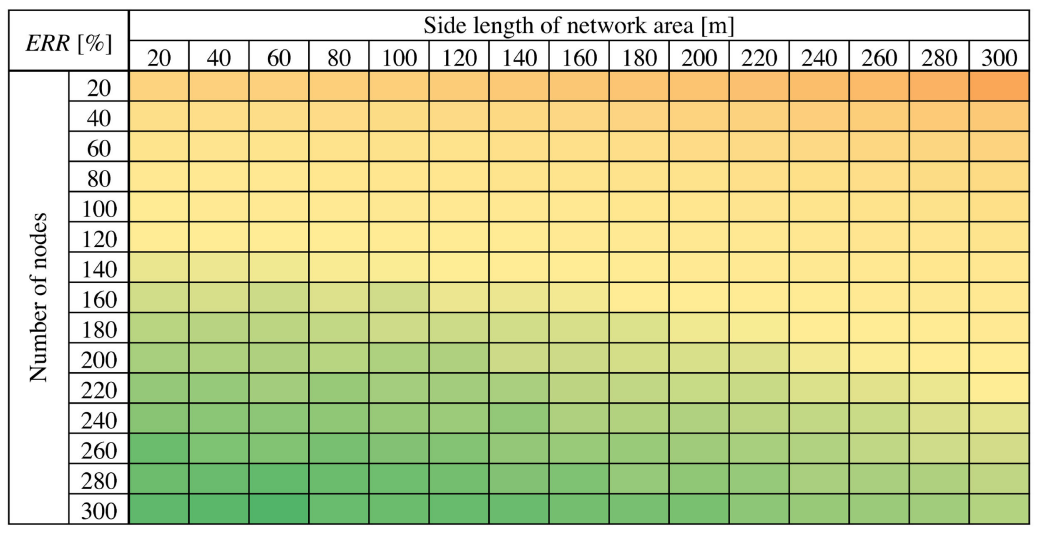

$30.2 \%$

$8.64 \%$

Fig. 2. A color map describing the energy reduction rate from SPT to PNF.

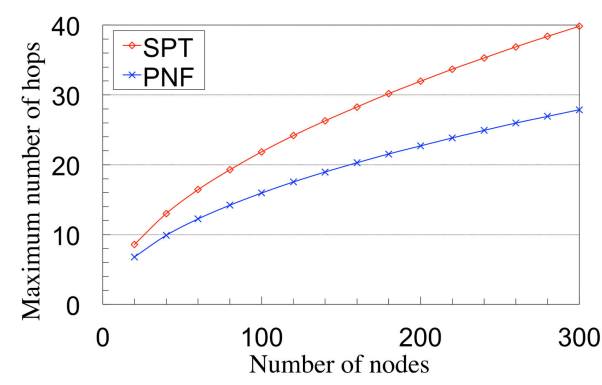

(a) Maximum number of hops

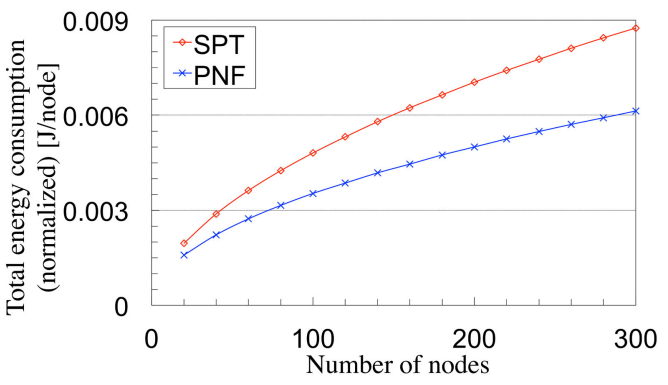

(b) Total energy consumption per node

Fig. 3. Maximum number of hops and total energy for SPT and PNF in a $100 \mathrm{~m} \times 100 \mathrm{~m}$ network.

increases in accordance with the number of nodes for both routing policies, PNF routing always results in the smaller hop-count than SPT. For a network with 20 and 300 nodes in a side length of $100 \mathrm{~m}$, the hop-count reduction rate is $20.9 \%$ and $30.0 \%$, respectively. PNF realizes short routes in terms of energy consumption by considering not only energy for data transmission but also hop progress, which leads to preventing its network routing from becoming detour paths. From these observations, it is clear that the hop-count reduction based on hop progress of PNF routing effectively reduces the energy cost for data forwarding.

\section{Conclusion}

This letter proposed a novel framework for energy-efficient routing policy in WSNs. The proposed method, named PNF routing, is based on variable-range TPC, nearest-neighbor routing, and progress toward a sink at each hop in order to reduce the extra number of hops. Performance evaluations showed that energy consumption in WSNs which employ PNF routing is less than SPT routing, with the energy reduction rate of at least $8.64 \%$ for the networks of interest. To further realize the network topology with minimum energy consumption, the forwarding angle for PNF routing is to be optimized. 